Mitteilungen aus dem Institut for angewandte Mathematics AN DER EIDGENÖSSISCHEN TECHNISCHEN HOCHSCHULE IN ZÜRICH HERAUSGEGEBEN VON PROF. DR. E. STIEFEL

Nr.7

\title{
Der Quotienten-Differenzen-Algorithmus
}

von

\section{Heinz Rutishauser}

Professor an der Eidgenössischen Technischen Hochschule in Zürich

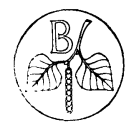

BIRKH ̈USER VERLAG BASEL/STUTTGART 
ISBN 978-3-7643-0323-5

ISBN 978-3-0348-7175-4 (eBook)

DOI 10.1007/978-3-0348-7175-4

Nachdruck verboten. Alle Rechte vorbehalten,

insbesondere das der Übersetzung in fremde Sprachen und der Reproduktion auf photostatischem Wege oder durch Mikrofilm

Birkhäuser Verlag Base1, 1957

(C) 


\section{NHALTS VERZ E I GH N I S ${ }^{1}$ )}

Einleitung. . . . . . . . . . . . . . . . . . . . . . 5

I. Kapitel. Theoretische Grundlagen

$\S$ 1. Problemstellung . . . . . . . . . . . . . . . . . . . . . 7

$\S$ 2. Der Quotienten-Differenzen-Algorithmus . . . . . . . . . . . . . . 8

$\S 3$. Die Rhombenregeln . . . . . . . . . . . . . . . . . . . . 10

$\S$ 4. Die zugeordneten Polynome $p_{\sigma}^{(v)}(z)$. . . . . . . . . . . . . 11

$\S$ 5. Beziehungen zur Kettenbruchtheorie . . . . . . . . . . . . 13

$\S$ 6. Schwierigkeiten bei der Bildung des QD-Schemas . . . . . . . . 16

$\S$ 7. Grundlegende Eigenschaften des QD-Algorithmus . . . . . . . 17

$\S$ 8. Beziehungen zum BO-Algorithmus von C. LANCzOs . . . . . . 20

$\S$ 9. Beziehungen zum cg-Algorithmus . . . . . . . . . . . . . . 22

$\S 10$. Ein Additionstheorem für Kettenbrüche . . . . . . . . . . . 23

II. Kapitel. Anwendungen des $Q D$-Algorithmus

$\S 1$. Umwandlung einer Potenzreihe in einen Kettenbruch . . . . . 26

$\S$ 2. Summation schlecht konvergenter Reihen . . . . . . . . . . . 27

$\S 3$. Auflösung von algebraischen Gleichungen . . . . . . . . . . . . . 29

$\S$ 4. Die progressive Form des QD-Algorithmus . . . . . . . . . . . . . 30

$\S 5$. Auflösung algebraischer Gleichung mit Hilfe des progressiven QD-Algorithmus . . . . . . . . . . . . . . . . . . . . . 31

$\S$ 6. Die Wronskische Formel . . . . . . . . . . . . . . . . . . . 33

$\S$ 7. Bestimmung komplexer Nullstellen . . . . . . . . . . . . . . . . . . . . 34

$\S$ 8. Quadratische Konvergenz des QD-Algorithmus . . . . . . . . 36

$\S$ 9. Massnahmen bei Division durch Null. . . . . . . . . . . . . 41

$\S$ 10. Interpolation durch Exponentialsummen . . . . . . . . . . . . . . 43

III. Kapitel. Bestimmung der Eigenwerte und Eigenvektoren einer Matrix mit Hilfe des Quotienten-Differenzen-Algorithmus

$\S$ 1. Die Bestimmung der Eigenwerte . . . . . . . . . . . . . . 49

$\S$ 2. Das Problem der Eigenvektorberechnung. . . . . . . . . . . . . . . . . . . 52

$\S 3$. Rekursive Berechnung der Vektoren $x_{0}^{(2 \mu)}, y_{\sigma}^{(2 \mu)}$. . . . . . . . . . 53

$\S$ 4. Ein quadratisch konvergentes Verfahren zur Eigenvektorbestimmung . . . . . . . . . . . . . . . . . . . . . . . . 59

$\S 5$. Eigenwerte und Eigenvektoren unendlicher symmetrischer Matrizen . . . . . . . . . . . . . . . 62

IV. Kapitel. Anhang

§ 1. Die LR-Transformation . . . . . . . . . . . . . . . . . . 65

$\S$ 2. Ein kontinuierliches Analogon zum QD-Algorithmus . . . . . . 69

$\S 3$. QD-Relaxation . . . . . . . . . . . . . . . . . . 73

Literaturverzeichnis . . . . . . . . . . . . . . . . . . 74

1) Bei Hinweisen im Text, die sich auf eine Formel oder einen Paragraphen eines andern Kapitels beziehen, wird die Nummer des betreffenden Kapitels beigefügt; zum Beispiel (II, 7) $=$ Formel 7 aus Kapitel II. 